\title{
Upregulation of Zip14 correlates with induction of endoplasmic reticulum stress (ERS) in hypertrophied hearts of Dahl salt- sensitive rats
}

\author{
Jinyong HUANG ${ }^{1}$; Tianming TENG ${ }^{1}$; Bo BIAN ${ }^{1}$; Yuchen XUE ${ }^{1}$; Longfei HUANG ${ }^{1}$; Zhelong XU ${ }^{2}$; QInG YANG ${ }^{1, *}$; \\ YUEMIN SUN ${ }^{1, *}$ \\ ${ }^{1}$ Department of Cardiology, Tianjin Medical University General Hospital, Tianjin, 300052, China \\ 2 Department of Physiology \& Pathophysiology, Tianjin Medical University, Tianjin, 300070, China
}

Key words: Zinc, Zip14, Endoplasmic reticulum stress (ERS), Left ventricular hypertrophy (LVH), Rat hearts

\begin{abstract}
Zinc is a trace element involved in maintaining cellular structure and function. Although zinc is associated with left ventricular hypertrophy (LVH), there have been few reports on this association. This study aimed to evaluate the correlation between Zip14 and expression of endoplasmic reticulum stress (ERS) associated molecules in hypertrophied hearts of rats. Dahl salt-sensitive rats were fed a high salt diet to establish a left ventricular hypertrophy (LVH) rat model. RT-PCR was used to determine Zip14, activating transcription factor (ATF4), ATF6, $\mathrm{x}$-box-binding protein 1 (xBP1), C/EBP homologous protein (CHOP), immunoglobulin-binding protein (BiP) mRNA expression. Western blotting was used to evaluate Zip14, BiP, CHOP, GAPDH expression. Zinc levels were measured by Inductively Coupled Plasma Optical Emission Spectroscopy. The results indicated that compared with the Control group, Zip14 mRNA and protein expression in LVH rat hearts were markedly increased $(P<0.01)$. Zinc content in rat heart tissue was significantly increased in the LVH group compared with the Control group $(P<0.05)$. ATF4, ATF6, xBP1 mRNA expressions were increased in LVH rat hearts compared with Control hearts $(P<0.001)$. Compared with the Control group, CHOP and BiP mRNA and protein expression were markedly increased in LVH rat hearts $(P<0.05, P<0.01)$. Linear regression models showed that Zip14 mRNA expressions were positively correlated with zinc concentration, ATF4 and ATF6 mRNA expressions in Control hearts $(P=0.0005, P=0.0052$, $P=0.0026$, respectively) and LVH rat hearts $(P<0.0001, P=0.0119, P=0.0033$, respectively). In conclusion, upregulation of Zip14 in LVH rat hearts correlated with zinc accumulation and induction of ERS.
\end{abstract}

\section{Introduction}

Zinc is a trace element involved in maintaining cellular structure and function (Ryul et al., 2015). High zinc levels have irreversible effects on proteins and lead to the dysfunction of many proteins. Low levels of zinc are also detrimental to cells because it is a cofactor for more than 300 enzymes and 2000 transcription factors, as well as mediating cell signaling (Roshanravan et al., 2015; Huang et al., 2017). Therefore, the balance of intracellular zinc concentration, termed zinc homeostasis, is critical. Under physiological conditions, the regulation of zinc homeostasis mainly depends on zinc transporters, zinc-binding

\footnotetext{
*Address correspondence to: Yuemin Sun, zyyxnksunyuemin@163.com; Qing Yang, cardio-yq@tmu.edu.cn

Received: 23 January 2021; Accepted: 08 March 2021
}

molecules, and zinc sensors (Foster and Samman, 2010). Zinc transporters are divided into two major families, Zip and ZnT. The 14 zinc transporters of the Zip family are responsible for transferring extracellular zinc into cells, while 10 zinc transporters of the $\mathrm{ZnT}$ family have the opposite role (Lichten and Cousins, 2009). Zip14 is located in the cell membrane and promotes extracellular zinc into the cytosol and increases the zinc concentration in the cytoplasm (Taylor et al., 2007).

The endoplasmic reticulum (ER) is an important organelle widely present in cells and an important site for the folding, assembly, and modification of protein molecules (Ron and Walter, 2007; Kim et al., 2008). When intracellular zinc is deficient, ER stress (ERS) occurs, causing dysfunction of the ER. Therefore, zinc is necessary to maintain normal ER function. In addition, ERS and cell dysfunction can be induced by oxidative stress and acute ischemia-reperfusion (IR) injury (Zhang, 2010; Zhang et al., 2014a; Zhang et al., 2014b). 
Zinc is associated with a variety of cardiovascular diseases, such as atherosclerosis and thrombosis of atherosclerotic plaque rupture, diabetic cardiomyopathy, arrhythmia, myocardial infarction, and congestive heart failure. Although zinc is associated with left ventricular hypertrophy (LVH), there have been few reports on this association (Little et al., 2010). LVH is characterized by pathological remodeling of the heart and is a good predictor of cardiovascular diseases such as myocardial infarction, congestive heart failure, sudden cardiac death, stroke, and overall CVD mortality (Desai et al., 2012). Our previous studies reported that serum zinc ion concentrations were significantly lower in patients with LVH compared with normal patients (Huang et al., 2017). Furthermore, the zinc trafficking and the activity of the Zip14 transporter are important for adapting to the ERSassociated chronic metabolic disorders, and the Zip14mediated transport of zinc is necessary for adapting ERS (Kim et al., 2017). Olgar et al. (2018a) also reported that $\mathrm{Zn}^{2+}$ correlates the induction of ERS through altering expressions of $\mathrm{Zn}^{2+}$-transporter, Zip14, in heart failure. Therefore, there might be a correlation between the Zip14 and the ERS. The present study aimed to investigate the mechanism of zinc transporter Zip14 and endoplasmic reticulum stress in the development of $\mathrm{LVH}$ in rats.

\section{Materials and Methods}

\section{Establishment of the LVH rat model}

To establish the LVH rat model, we chose Dahl salt-sensitive rats and fed them a high salt diet. The control group was fed a normal salt diet. The content of sodium chloride in the high salt feed was $8 \%$, while the normal diet group contained $0.3 \%$. We purchased 5-week-old rats of SPF grade from Beijing Weitong Lihua Experimental Animal Technology Co. Ltd., (license key SCXK (Beijing) 2012-0001). Blood pressure and heart rate were measured by a noninvasive blood pressure detector (BP-2010AUL Softron, Tokyo, Japan) every two weeks. All animals were exposed to a 12-h light-dark cycle and were given free access to tap water and standard chow daily.

The rats in this study were randomly divided into the Control group ( $\mathrm{N}=14,7$ males and 7 females) and $\mathrm{LVH}$ group $(\mathrm{N}=22,11$ males and 11 females). At weeks 6, 12, and 18 , rats were anesthetized by the intraperitoneal injection of $30 \mathrm{mg} / \mathrm{kg} \mathrm{10 \%} \mathrm{chloral} \mathrm{hydrate.} \mathrm{Then,} \mathrm{the} \mathrm{rats} \mathrm{were} \mathrm{fixed} \mathrm{in}$ a supine position, excluding the neck and chest hair, and coated with appropriate coupling agents. Mild sedation was maintained through a nasal tube with a continuous low flow of oxygen. The heart rate was maintained at about 300-350 bpm and was detected by an animal-specific ultrasound system (VEVO 2100 Imaging System, Toronto, Canada). LVH was successfully induced in high salt-fed Dahl rats at about 18 weeks. Left ventricular myocardium specimens were removed from successful LVH model rats and the Control group at various time points, immediately frozen in liquid nitrogen, and stored at $-80^{\circ} \mathrm{C}$ until use.

RT-PCR assay

Total RNA was extracted from myocardial tissues using Trizol Reagent (Invitrogen) following the manufacturer's recommendations. RNA concentrations were determined by

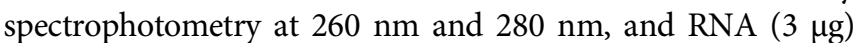
was reverse transcribed to obtain complementary DNA (cDNA) in a $20 \mu \mathrm{L}$ reaction mixture with Promega Reverse Transcription System (Sigma-Aldrich.) according to manufacturer recommendations. Primers used for RT-PCR amplification were designed with Software Primer 5.0 and synthesized by Invitrogen Company (Beijing, China). Realtime PCR was performed with the primer sequences listed in Table 1 using a CFX96 Real-Time PCR System (Bio-Rad, Singapore) and a SYBR $^{\odot}$ Green PCR Kit (Invitrogen) according to a standard application protocol and the manufacturer's instructions. The cycling parameters were as follows: $95^{\circ} \mathrm{C}$ for $10 \mathrm{~min}, 40$ cycles of $95^{\circ} \mathrm{C}$ for $15 \mathrm{~s}$, and the annealing/extension temperature and time is $60^{\circ} \mathrm{C}$ for $60 \mathrm{~s}$. All samples were assayed in triplicate. The control gene GAPDH RNA was used to normalize the results. The comparative threshold cycle method was used for data analyses.

\section{Western blotting analysis}

Myocardial samples taken from the left ventricular region were homogenized in ice-cold lysis buffer. The protein concentrations of supernatants after centrifugation (15000 $\mathrm{rpm}, 5 \mathrm{~min}$, at $4^{\circ} \mathrm{C}$ ) were measured with the BCA assay kit (Pierce) according to the manufacturer's instructions. Equal amounts of cytosolic protein were loaded and electrophoresed on $10 \%$ SDS-polyacrylamide gel and transferred to a PVDF membrane. Membranes were blocked in 5\% nonfat milk and then incubated with primary antibodies diluted in 4\% BSA in PBS-Tween at $4^{\circ} \mathrm{C}$ overnight. The membranes were probed with antibodies against Zip14 (oriGene, TA319861; 1:1500, poly), immunoglobulin-binding protein (BiP) (ImmunoWay, YM3508; 1:1000, mono), C/EBP homologous protein (CHOP) (Proteintech, 15204-I-AP, 1:500, poly), and GAPDH

\section{TABLE 1}

Primer sequences used for quantitative real-time RT-PCR

\begin{tabular}{lll}
\hline Gene & & Primer sequences (5' ${ }^{\prime}{ }^{\prime}$ ) \\
\hline \multirow{2}{*}{ Zip14 } & F & TCAGCCGTGTGCTCACTTAC \\
& R & GTCCTGAGGGTTGAAACCAA \\
ATF4 & F & AAACCTCATGGGTTCTCCAG \\
& R & GGTTTCCAGGTCATCCATTC \\
ATF6 & F & GAGAGGTGTCTGTTTCGGGG \\
& R & CGTGGACTCCCAGTCTTCAC \\
xBP1 & F & GGATTTGGAAGAAGAGAACCA \\
& R & TTCAGTAACCAGGGCATTCA \\
CHOP & F & CACACCTGAAAGCAGAAACC \\
& R & GACCTCCTGCAGATCCTCAT \\
BiP & F & ATCATCAATGAGCCAACAGC \\
& R & TTAGTGGCCACCACTTCAAA \\
GAPDH & F & GGTGATGCTGGTGCTGAGTA \\
& R & ACTGTGGTCATGAGCCCTTC \\
\hline
\end{tabular}

Note: F: forward; R: reverse; ATF4: activating transcription factor 4; ATF6: activating transcription factor 6 ; $\mathrm{xBP} 1$ : $\mathrm{x}$ box-bindingprotein 1 ; $\mathrm{CHOP}$ : $\mathrm{C} / \mathrm{EBP}$ homologous protein; $\mathrm{BiP}$ : immunoglobulin-binding protein. 
(CST, 2118S; 1:1500, mono) in BSA/PBS/Tween 20 solution. The binding of the primary antibody was detected with secondary antibodies (anti-rabbit, 1:2500) and visualized by the ECL method. The intensities of the bands were analyzed using Image J software.

\section{Measurement of zinc concentrations}

Fifty mg myocardial tissue and $1 \mathrm{~mL}$ of $65 \%$ concentrated nitric acid was added to a small beaker for digestion and placed on a graphite-controlled thermoelectric plate at $120^{\circ} \mathrm{C}$ for $30 \mathrm{~min}$. Finally, the nitric acid volume was made to $1 \mathrm{~mL}$, and $4 \mathrm{~mL}$ of distilled water was added. The zinc levels were measured by Inductively Coupled Plasma Optical Emission Spectroscopy (ICP-OES optima 8300, Perkin Elmer, MA, USA).

\section{Statistical analysis}

Data are expressed as the mean \pm SEM and were obtained from 6 to 10 separate experiments. Statistical analysis of experimental data was performed using SPSS 22.0 software (IBM Corp., Armonk, NY, USA). Statistical significance was determined using the Student's $t$-test or repeated-measures analysis of variance test. Linear regression analysis was used for relationships between variables. A value of $P<0.05$ was considered statistically significant.

\section{Results}

Hemodynamic and echocardiographic parameters of rats at different ages

The parameters of the rats are presented in Table 2. There was no significant difference in each index between LVH and Control group rats at week 6 . The mortality rate of the LVH group rats was $22.7 \%$ from weeks 6 to 12 .

The mean systolic blood pressure (SBP) and late systolic thickness of the posterior wall of the left ventricle (LVPWs) in the LVH group were significantly higher than in the Control group (respectively, $P<0.05$ ). However, the body weight (BW) was lower in the LVH group than in the Control group $(P<0.05)$ at week 12 .

The mean systolic blood pressure (SBP) and mean diastolic blood pressure (DBP) were significantly increased in the LVH group compared with the Control group (respectively, $P<0.05$ ) at week 18 . However, the SBP arose in 12-week and 18-week-old rats in the Control group, which might be caused by the increased body weight or obesity. The interventricular septum thickness at enddiastole (IVSd), left ventricular end-diastolic diameter (LVDd), the end-diastolic thickness of the left ventricular wall (LVPWd), and late systolic thickness of the posterior wall of the left ventricle (LVPWs) in the LVH group were significantly higher than in the Control group (all $P<0.05$ ). However, body weight (BW) was lower in the LVH group compared with the Control group $(P<0.05)$ at week 18 .

\section{Successful establishment of a LVH rat model}

As shown in Fig. 1, the left ventricular mass (LVM) and left ventricular hypertrophy index (LVM/BW) were significantly higher in the LVH group compared with the Control group at week $18(744.83 \pm 104.74 v s .635 .83 \pm 119.06 \mathrm{mg}, 2.29 \pm$ 0.34 vs. $1.56 \pm 0.32$, respectively, $P<0.05)$. In addition to being a LVH rat model, it is also a classic rat model of hypertension.

Expression of Zip14 and zinc contents are increased in LVH rat hearts

As shown in Fig. 2A, Zip14 mRNA expression was markedly higher in LVH compared with Control rats $(P<0.01)$. A representative western blotting band for Zip14 is shown in Fig. 2B. Western blot analysis demonstrated that the expression of Zip14 proteins was increased in the LVH rat heart compared with Control hearts $(P<0.01)$ (Fig. 2B). In

TABLE 2

Fundamental and echocardiographic data for rats of different periods

\begin{tabular}{|c|c|c|c|c|c|c|}
\hline \multirow{2}{*}{$\begin{array}{l}\text { Age (week) } \\
\text { Groups }\end{array}$} & \multicolumn{2}{|c|}{6} & \multicolumn{2}{|c|}{12} & \multicolumn{2}{|c|}{18} \\
\hline & Control & $\mathrm{LVH}$ & Control & LVH & Control & LVH \\
\hline $\mathrm{N}$ & 14 & 22 & 14 & 17 & 14 & 17 \\
\hline SBP (mmHg) & $114.54 \pm 14.19$ & $116.23 \pm 13.36$ & $131.21 \pm 4.85^{\mathrm{a}}$ & $144.88 \pm 10.28^{\star a}$ & $133.33 \pm 3.78^{\mathrm{a}}$ & $168.73 \pm 7.62^{\star a b}$ \\
\hline $\mathrm{DBP}(\mathrm{mmHg})$ & $85.83 \pm 16.99$ & $84.88 \pm 10.52$ & $100.38 \pm 5.71^{\mathrm{a}}$ & $104.45 \pm 9.77^{\mathrm{a}}$ & $102.54 \pm 5.08^{\mathrm{a}}$ & $113.57 \pm 7.73^{\star a}$ \\
\hline HR (bpm) & $361.75 \pm 41.52$ & $370.50 \pm 27.59$ & $409.04 \pm 7.41$ & $399.76 \pm 15.36$ & $412.33 \pm 7.23$ & $400.31 \pm 17.00$ \\
\hline IVSd (mm) & $1.83 \pm 0.28$ & $1.81 \pm 0.32$ & $1.96 \pm 0.18$ & $2.16 \pm 0.28$ & $2.01 \pm 0.18$ & $2.33 \pm 0.32^{\star a}$ \\
\hline LVDd (mm) & $6.07 \pm 0.36$ & $6.09 \pm 0.73$ & $6.21 \pm 0.21$ & $6.48 \pm 0.59$ & $6.26 \pm 0.30$ & $6.75 \pm 0.53^{\star a}$ \\
\hline LVDs (mm) & $2.71 \pm 0.50$ & $2.81 \pm 0.49$ & $2.94 \pm 0.50$ & $3.22 \pm 0.48$ & $3.03 \pm 0.55$ & $3.41 \pm 0.52$ \\
\hline LVPWd (mm) & $1.79 \pm 0.31$ & $1.80 \pm 0.17$ & $1.88 \pm 0.21$ & $2.05 \pm 0.16$ & $1.92 \pm 0.26$ & $2.16 \pm 0.23^{\star a}$ \\
\hline LVPWs (mm) & $2.68 \pm 0.32$ & $2.71 \pm 0.26$ & $2.80 \pm 0.15$ & $3.09 \pm 0.12^{\star a}$ & $2.99 \pm 0.12$ & $3.27 \pm 0.21^{\star a}$ \\
\hline $\mathrm{EF}(\%)$ & $77.71 \pm 9.71$ & $79.84 \pm 7.82$ & $75.31 \pm 6.37$ & $75.93 \pm 6.50$ & $74.10 \pm 7.23$ & $71.50 \pm 7.79$ \\
\hline $\mathrm{BW}(\mathrm{g})$ & $179.63 \pm 7.09$ & $177.83 \pm 12.25$ & $333.25 \pm 9.11^{\mathrm{a}}$ & $275.08 \pm 4.38^{\star \mathrm{a}}$ & $410.12 \pm 12.64^{\mathrm{ab}}$ & $323.58 \pm 9.59^{* a b}$ \\
\hline
\end{tabular}

Note: ${ }^{\star} v s$. Control group $P<0.05 ;{ }^{\mathrm{a}} v s$. week $6 P<0.05 ;{ }^{\mathrm{b}}$ vs. week $12 P<0.05$. SBP: systolic blood pressure; DBP: diastolic blood pressure; HR: heart rate; IVSd: interventricular septum thickness at end-diastole; LVDd: left ventricular end-diastolic diameter; LVDs: left ventricular end-systolic diameter; LVPWd: enddiastolic thickness of the left ventricular wall; LVPWs: late systolic thickness of the posterior wall of the left ventricle; EF: ejection fraction; BW: body weight. 
A LVM

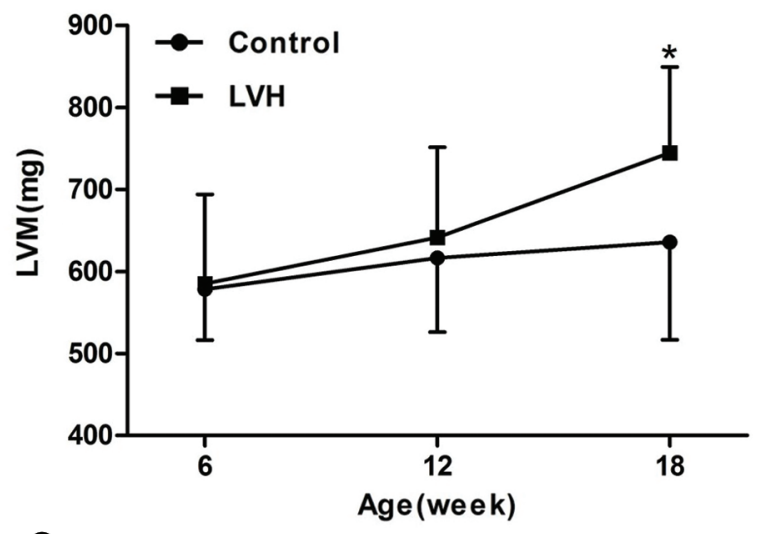

C

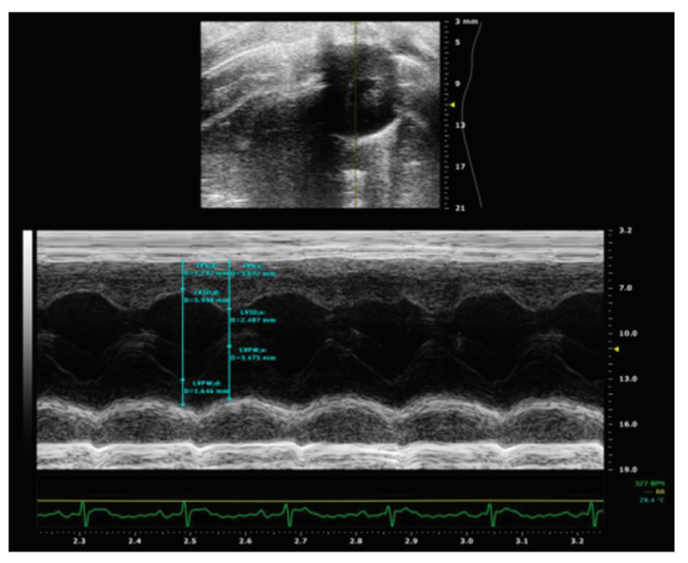

B LVM/BW
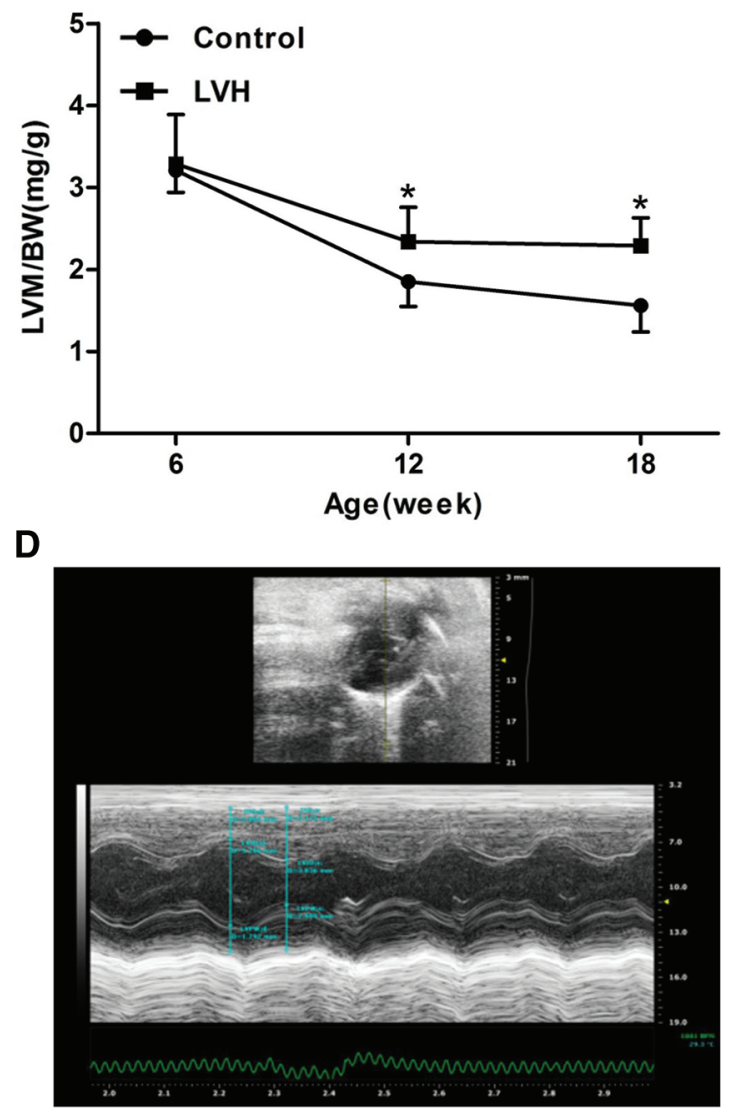

FIGURE 1. Establishment of left ventricular hypertrophy $(\mathrm{LVH})$ rat models $(\mathrm{N}=10)$. (A) The left ventricular mass $(\mathrm{LVM})$ in the LVH heart was increased compared with the Control group at the $18^{\text {th }}$ week $(744.83 \pm 104.74 v s .635 .83 \pm 119.06 \mathrm{mg}),{ }^{\star} P<0.05$ vs. Control group. (B) The left ventricular hypertrophy index (LVM/BW) in the LVH heart was increased compared with the Control group at the $18^{\text {th }}$ week $(2.29 \pm 0.34$ vs. $1.56 \pm 0.32),{ }^{\star} P<0.05$ vs. Control group. (C) Representative transverse axial echocardiographic image of the control rat heart. (D) Representative transverse axial echocardiographic image of the LVH rat heart.
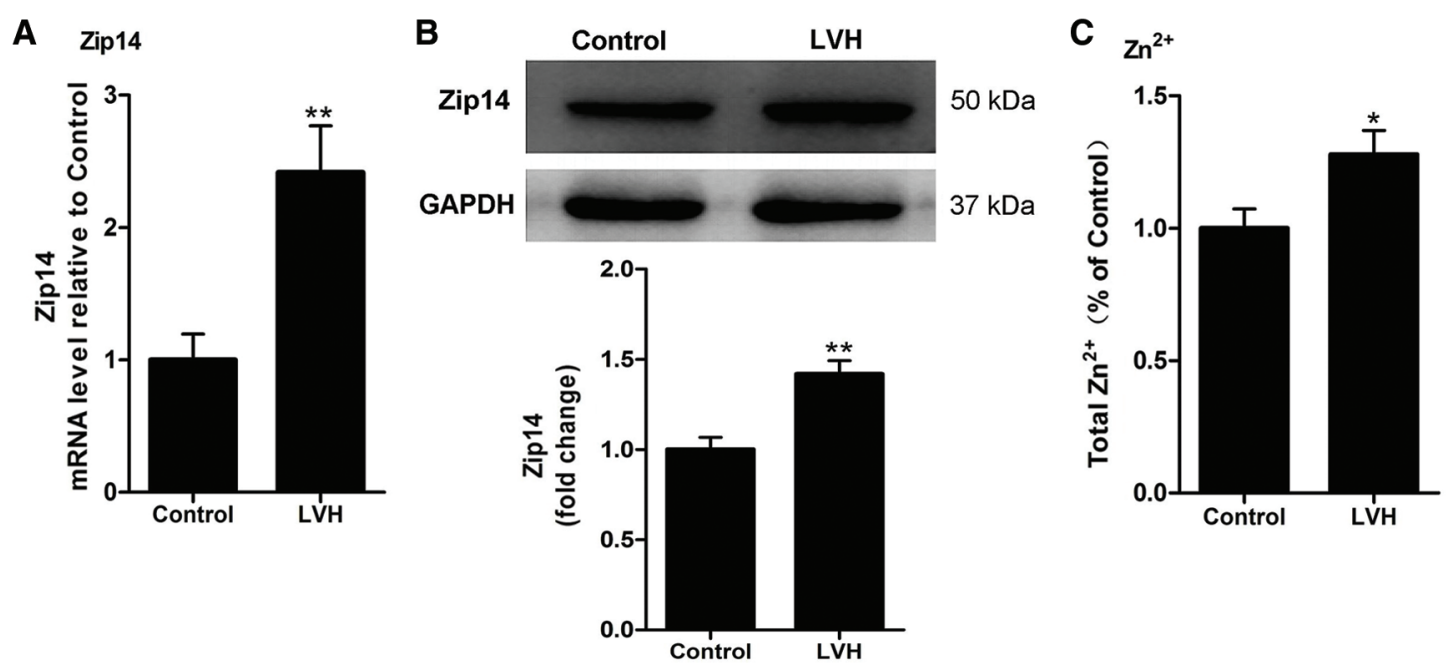

FIGURE 2. Expression of Zip14 and $\mathrm{Zn}^{2+}$ contents in rat hearts. (A) Relative mRNA expression level of Zip14 in rat hearts $(\mathrm{N}=10)$, ${ }^{* *} P<0.01$ $v s$. Control group. (B) Representative Western blotting images and statistical analysis $(\mathrm{N}=6)$ showing the expression of Zip14 proteins in rat hearts. ${ }^{* *} P<0.01$ vs. Control group. (C) The $\mathrm{Zn}^{2+}$ contents in the LVH heart were increased compared with Control group (1.28 $\pm 0.29 v s .1 .00$ $\pm 0.23 \mathrm{mg} / \mathrm{L}, \mathrm{N}=10),{ }^{\star} P<0.05$ vs. Control group.

addition, as shown in Fig. 2C, the zinc concentration was significantly increased in LVH rat hearts compared with Control hearts $(1.28 \pm 0.29 v s .1 .00 \pm 0.23 \mathrm{mg} / \mathrm{L}, P<0.05)$.
Induction of ERS in LVH rat hearts

When ERS occurs, plenty of molecules are involved in this process. Therefore, to demonstrate the occurrence of ERS, 
we detected signal transduction molecules by western blotting and RT-PCR. As shown in Figs. 3A-3C, the mRNA expressions of activating transcription factor 4 (ATF4), activating transcription factor 6 (ATF6), and $\mathrm{x}$ box-binding protein $1(\mathrm{xBP} 1)$ were increased in the $\mathrm{LVH}$ rat heart compared with Control hearts $(P<0.001)$.

As shown in Fig. 3D, CHOP mRNA expression was markedly higher in LVH hearts compared with Control hearts $(P<0.001)$. A representative western blotting band for CHOP is shown in Fig. 3E. Western blot analysis demonstrated that the expression of CHOP proteins was increased in the $\mathrm{LVH}$ rat heart compared with Control hearts $(P<0.05)$ (Fig. 3E). As shown in Fig. 3F, BiP mRNA expression was markedly higher in LVH hearts compared with Control hearts $(P<0.05)$. A representative western blotting band for $\mathrm{BiP}$ is shown in Fig. 3G. Western blot analysis demonstrated that the expression of $\mathrm{BiP}$ proteins was increased in the $\mathrm{LVH}$ rat heart compared with Control hearts $(P<0.05)$ (Fig. 3G).

Zip14 mRNA expressions are positively correlated with zinc contents, ATF4, and ATF6 mRNA expression

To confirm the relationship between Zip14 and zinc, ATF4, and ATF6, we performed linear regression analyses. As shown in
Figs. $4 \mathrm{~A}$ and $4 \mathrm{~B}$, the linear regression models showed a significant positive relationship between Zip14 mRNA expressions and zinc concentration in Control hearts $\left(R^{2}=\right.$ $0.8027, P=0.0005)$ and LVH rat hearts $\left(R^{2}=0.8769, P<0.0001\right)$.

Figs. $4 \mathrm{C}$ and $4 \mathrm{D}$ showed the positive relationships between Zip14 mRNA expressions and ATF4 mRNA expressions in the Control $\left(R^{2}=0.6436, P=0.0052\right)$ and LVH groups $\left(R^{2}=0.5674, P=0.0119\right)$. As shown in Figs. 4E-4F, Zip14 mRNA expressions were positively correlated with ATF6 in Control $\left(R^{2}=0.6978, P=0.0026\right)$ and LVH $\left(R^{2}=0.6807, P=0.0033\right)$ rat hearts.

\section{Discussion}

Our research provides new and interesting insights into the complex relationship between zinc and LVH. A model of LVH by feeding Wistar germline Dahl salt-sensitive rats a high salt diet has been successfully established. At week 18, the left ventricular mass and left ventricular hypertrophy index of the experimental group were significantly higher than in the control group. We also detected a marked increase in zinc concentration and a remarkable upregulation of zinc transporter Zip14 expression in
A ATF4

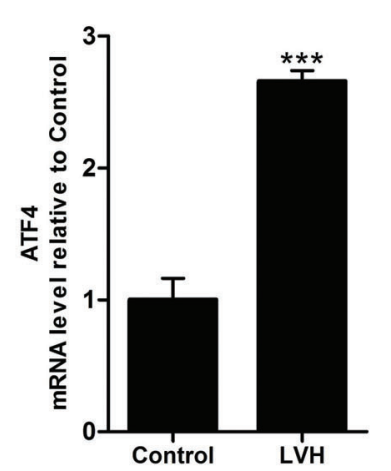

B

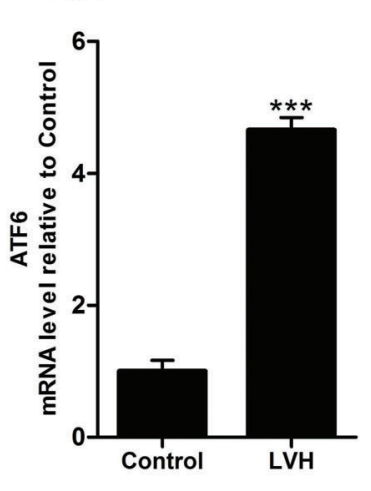

C $\quad x B P 1$

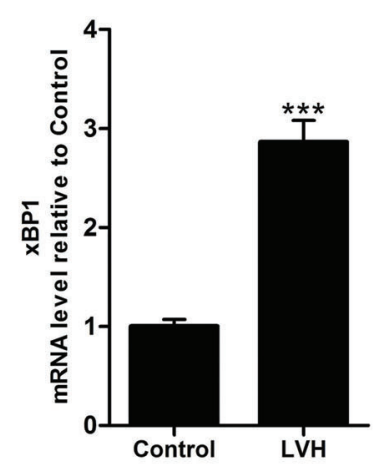

D

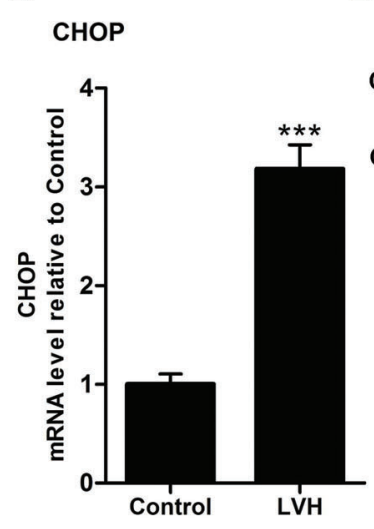

E

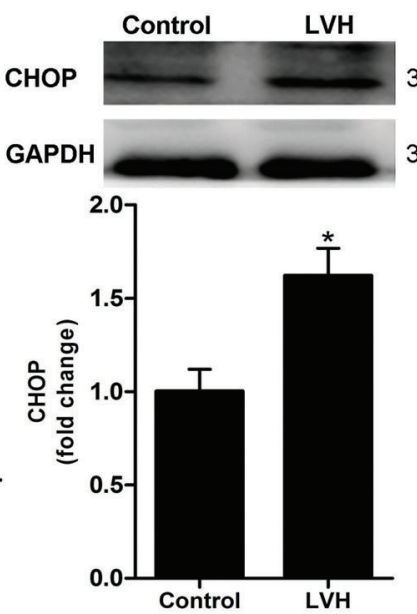

$\mathbf{F}$

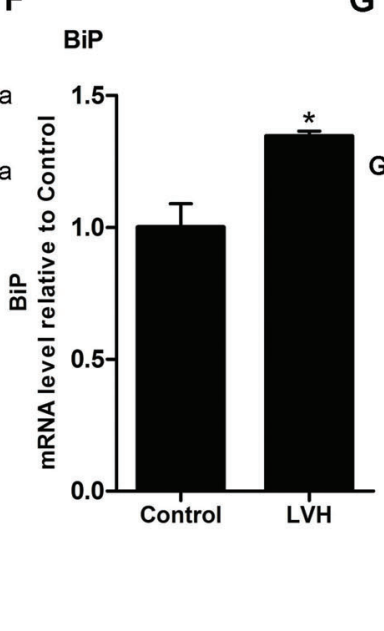

$\mathbf{G}$

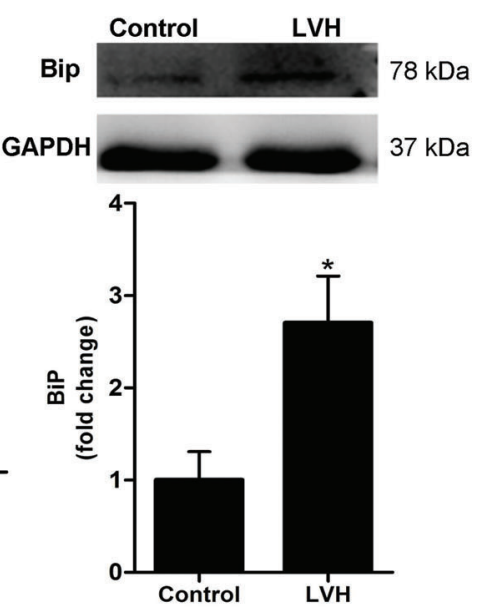

FIGURE 3. Induction of endoplasmic reticulum stress (ERS) in rat hearts. (A) Relative mRNA expression level of ATF4 in rat hearts ( $N=10$ ), ${ }^{* * *} P<0.001 v s$. Control group. (B) Relative mRNA expression level of ATF6 in rat hearts $(\mathrm{N}=10),{ }^{* * *} P<0.001$ vs. Control group. (C) Relative mRNA expression level of xBP1 in rat hearts $(\mathrm{N}=10),{ }^{* \star \star} P<0.001 v$ s. Control group. (D) Relative mRNA expression level of CHOP in rat hearts $(\mathrm{N}=10),{ }^{* *} P<0.001$ vs. Control group. (E) Representative Western blotting images and statistical analysis $(\mathrm{N}=6)$ showing the expression of $\mathrm{CHOP}$ proteins in rat hearts. ${ }^{\star} P<0.05 v$ s. Control group. (F) Relative mRNA expression level of BiP in rat hearts $(\mathrm{N}=10)$, ${ }^{*} P<0.05$ vs. Control group. $(\mathrm{G})$ Representative Western blotting images and statistical analysis $(\mathrm{N}=7)$ showing the expression of $\mathrm{BiP}$ proteins in rat hearts. ${ }^{\star} P<0.05 v$ s. Control group. 
myocardial tissues of LVH rats. Furthermore, the mRNA expressions of ATF4, ATF6, CHOP, BiP, and xBP1 in LVH rats under ERS were significantly upregulated and partially related to $\mathrm{CHOP}$ protein levels in the myocardial tissues of $\mathrm{LVH}$ rats. A marked increase in the expression of $\mathrm{BiP}$ confirmed the occurrence of ERS in the myocardial tissues of LVH rats.

Zinc plays an important role in cardiomyocyte protection by involving lots of signaling pathways, such as the cGMP/PKG pathway (Jang et al., 2007), NO/cGMP/PKG and glycogen synthase kinase-3 $\beta$ (GSK-3 $\beta$ ) signaling pathway (Xi et al., 2010). Moreover, the zinc transporter
Zip14 is closely related to inflammation and production of proinflammatory cytokines (Aydemir et al., 2012; Eizirik et al., 2012; Troche et al., 2016). Zip14 is also expressed in cardiac tissues and located in the plasma membrane, which promotes extracellular zinc into the cytosol and increases the concentration of zinc in the cytosol (Taylor et al., 2007; Olgar et al., 2018a). We observed higher expression levels of Zip14 and ICP-measured myocardial tissue zinc ions in the LVH rat model compared with the Control group. Similarly, Olgar et al. (2018b) used a rat hypertrophic heart model induced by transverse aortic coarctation (TAC) to show that the expression of Zip14 and the concentration of zinc ions
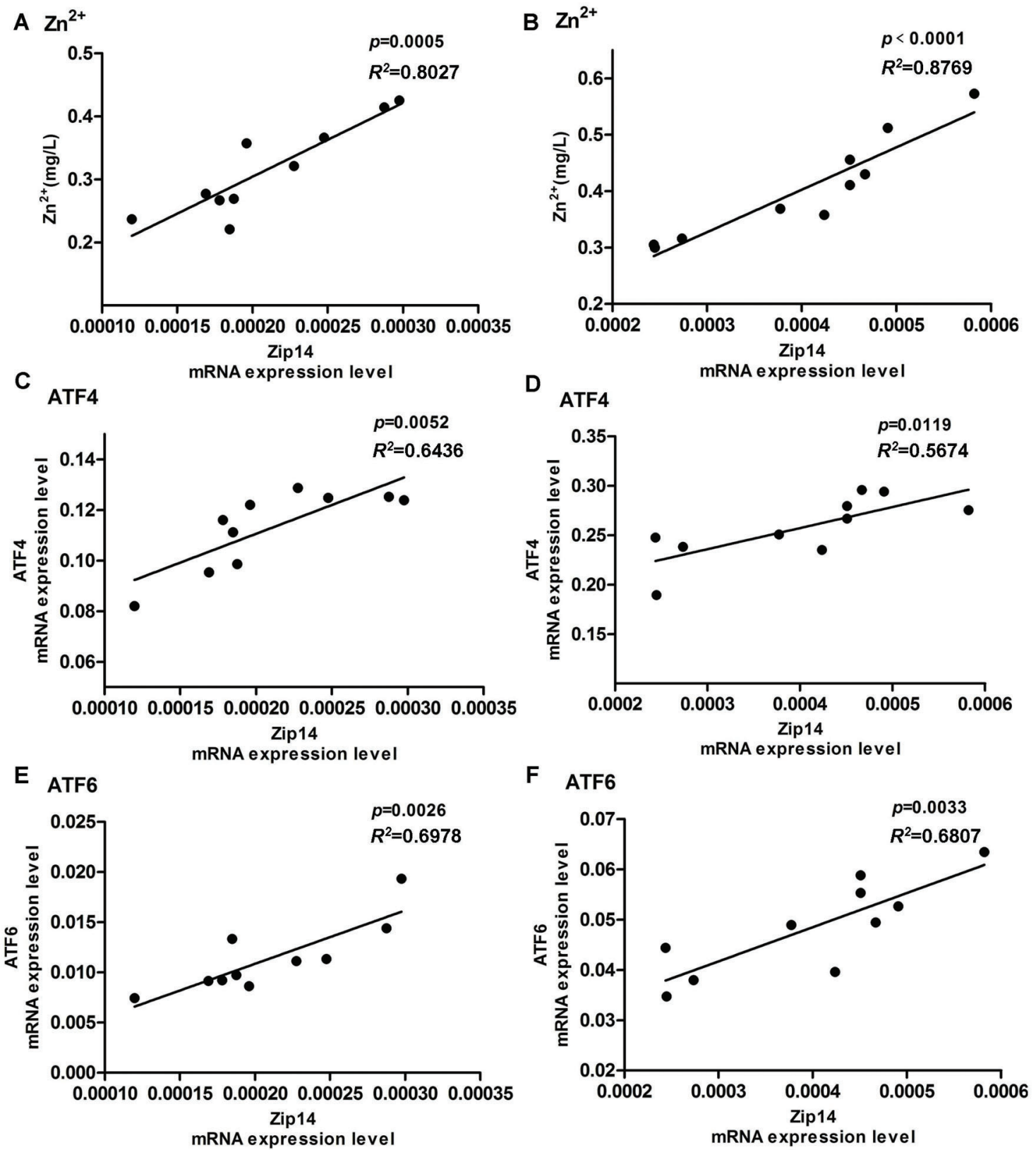

FIGURE 4. Linear regression analysis. (A) Linear regression analysis between Zip14 mRNA expression level and $\mathrm{Zn}^{2+}$ concentration in rat hearts of Control group $\left(\mathrm{Zn}^{2+}=1170.5 \times \mathrm{Zip} 14+0.0701, R^{2}=0.8027\right)$. (B) Linear regression analysis between Zip14 mRNA expression level and $\mathrm{Zn}^{2+}$ in LVH rat hearts $\left(\mathrm{Zn}^{2+}=752.45 \times \mathrm{Zip} 14+0.1017, R^{2}=0.8769\right)$. (C) Linear regression analysis of mRNA expression level between Zip14 and ATF4 in rat hearts of Control group (ATF4 $=227.76 \times \mathrm{Zip} 14+0.065, R^{2}=0.6436$ ). (D) Linear regression analysis of mRNA expression level between Zip14 and ATF4 in LVH rat hearts (ATF4 $=213.15 \times$ Zip14 $\left.+0.1719, R^{2}=0.5674\right)$. (E) Linear regression analysis of mRNA expression level between Zip14 and ATF6 in rat hearts of Control group (ATF6 $=53.194 \times$ Zip14 $+0.0002, R^{2}=0.6978$ ). (F) Linear regression analysis of mRNA expression level between Zip14 and ATF6 in LVH rat hearts $\left(\right.$ ATF6 $=67.922 \times$ Zip14 $\left.+0.0213, R^{2}=0.6807\right)$. 
in cardiomyocytes were increased compared with the SHAM group. We found that Zip14 plays an important role in the increased zinc concentration in the myocardium of $\mathrm{LVH}$ rats through linear regression analysis. ERS occurs when cells are affected by external factors such as inflammation, oxidative stress, and intracellular zinc homeostasis. ERS refers to misfolding and folding disorders of newly synthesized proteins leading to unfolded and misfolded proteins accumulating in the ER, affecting the normal function of the ER (Schroder and Kaufman, 2005; Kim et al., 2017). Activation of the UPR-related signaling pathway increased the expression of ER chaperones such as $\mathrm{BiP}$ and induced apoptosis independently (Walter and Ron, 2011); therefore, we determined the expression of Bip molecule in our study. Recently, Kim et al. (2017) confirmed that Zip14 is closely related to the adaptation of ER to livermediated stress and reduces hepatic steatosis and apoptosis by activating the ATF4 and ATF6 $\alpha$ and UPR reactions (Kim et al., 2017). Therefore, when ERS occurs in cells, increased intracellular $\mathrm{Zn}^{2+}$ concentrations play an important role in cell protection. Our findings showed that expressions of ATF4, ATF6, xBP1, and BiP were increased in LVH rat hearts compared with Control hearts. Meanwhile, ERS were significantly upregulated and partially related to CHOP protein levels in the myocardial tissues of LVH rats. All these results suggest that Zip14 is associated with the ERS in the LVH rat models. Moreover, according to the previous studies, many key biomarkers (Rutkowski et al., 2006; Wang et al., 2016) (such as PKR-like ER 1 kinase (PERK), eukaryotic initiation factor $2 \alpha(\mathrm{eIF} 2 \alpha)$ ) and signaling pathways (such as p-eIF2a/ATF4/CHOP pathway) (Rutkowski et al., 2006), also involve in ERS and UPR. Meanwhile, the $\mathrm{Zn}^{2+}$ participated metabolism of cardiomyocytes is also associated with the ERS, which involving Zip14, Zip7, and other molecules (MacDonald, 2000; Yoshida, 2007; Murakami and Hirano, 2008; Wang et al., 2016; Tuncay et al., 2017; Xing et al., 2017). Therefore, the association between Zip14 (or other $\mathrm{Zn}^{2}$ ${ }^{+}$-transporters) and ERS in LVH animal models should be verified in future investigations.

Furthermore, linear regression analysis indicated that the expression of Zip14 mRNA expression in the myocardial tissues of the Control and experimental groups was positively correlated with zinc concentration. The mRNA expressions of Zip14, ATF4, and ATF6 in the myocardial tissues of the Control and experimental groups were also positively correlated. However, the correlation between Zip14 expression and $\mathrm{CHOP}$ or $\mathrm{BiP}$ has not been verified in our study, which is needed to be detected in further studies.

Therefore, we hypothesized that ERS in the myocardium of LVH rats induced by external factors such as inflammation and oxidative stress upregulates the expression of ATF4 and ATF6 by activating UPR-related pathways or the activation of other pathways to increase the expression of Zip14 to increase the zinc concentration in cardiomyocytes. A shortterm increase in zinc concentration allows cells to adapt to ERS and protects cardiomyocytes. Sustained ERS and the long-term increase of the zinc concentration in cardiomyocytes may cause myocardial cell apoptosis and dysfunction, which might be a pathophysiological mechanism in the development of $\mathrm{LVH}$.
There were some limitations in this study. Firstly, in the process of studying how Zip14 regulates zinc homeostasis, there was no intervention study of zinc and Zip14; thus, it was not clear which was the originating factor. To clarify the role of zinc in the pathogenesis of LVH, we will establish Zip14 gene knockout LVH rats fed with different concentrations of zinc and will use the ER inhibitor TUDCA to investigate the relationship between the Zip14 regulation of zinc homeostasis and ERS. Also, we would examine whether the ERS is inhibited by zinc supplementation in the $\mathrm{LVH}$ rat model in the following studies. Secondarily, this study mainly focused on the correlation between Zip14 and ERS induction of LVH animal model; however, the unfolded protein response (UPR) pathway and associated sensors, such as IRE phosphorylation, PERK (or eIF2 $\alpha$ ) phosphorylation or ATF6 cleavage, have not been determined here. In a future study, we would determine more contents such as PERK/eIF2 $\alpha$, cleaved ATF6, spliced XBP1 signaling pathways and associated molecules to enrich the findings and conclusions. Thirdly, the link between ERS and Zip upregulation has not been clearly investigated in this study. A previous study reported that under pharmacologically induced ERS or chemically alleviated protein misfolding, the Zip14 and zinc content are upregulated (Kim et al., 2017). Therefore, knocking down the Zip14 gene, such as siRNA targeting, would be better for clarifying the link between Zip14 and ERS. Meanwhile, the potential common transcription factor binding sites (TFBS) clusters in the promoter regions of gene Zip14, ATF6, ATF4 might be significant for demonstrating the correlation between Zip14 and ATF6 or ATF4. Fourthly, in the process of modeling, the body weight of rats was lower in LVH group compared to the Control group, which is another limitation of our study. We speculated that the body weight loss might be caused by the treatment of high salt fed (might affect the appetite of the rats). Finally, the $\mathrm{Zn}^{2+}$ contents might affect the effects of Zip14; however, $\mathrm{Zn}^{2+}$ contents in the blood of rats in both groups have not been determined.

\section{Conclusions}

Zinc accumulation and the upregulation of Zip14 and ERS were observed in myocardial tissues of rats with LVH. The upregulation of Zip14 in LVH rat hearts correlated with zinc accumulation and induction of ERS. However, the exact mechanism of these interactions needs to be investigated further.

Acknowledgement: We thank the support and help of the team of Professor Xu Zhelong from the School of Basic Medicine, Tianjin Medical University.

Availability of Data and Materials: All data generated or analyzed during this study are included in this published article (and its supplementary information files).

Author Contribution: The authors confirm contribution to the paper as follows: study conception and design: QY and YS; data collection: JH, TT, BB, YX, LH, ZX; analysis and interpretation of results: JH, LH, ZX, YS; draft manuscript 
preparation: JH, QY and YS. All authors reviewed the results and approved the final version of the manuscript.

Ethics Approval: All animal treatments were strictly in accordance with international ethical guidelines and the National Institute of Health Guide concerning the Care and Use of Laboratory Animals. The experiments were carried out with the approval of the Committee of Experimental Animal Administration of the University (ethical approval code: ZYY-IRB-SOP-016(F)-002-02, date of approval: 30th, April, 2015).

Funding Statement: This work was supported by the key projects of Tianjin Natural Science Foundation (Grant No. 17JCZDJC34800).

Conflicts of Interest: The authors declare that they have no conflicts of interest to report regarding the present study.

\section{References}

Aydemir TB, Chang SM, Guthrie GJ, Maki AB, Ryu MS, Karabiyik A, Cousins RJ (2012). Zinc transporter ZIP14 functions in hepatic zinc, iron and glucose homeostasis during the innate immune response (endotoxemia). PLoS One 7: e48679. DOI 10.1371/journal.pone.0048679.

Desai CS, Ning H, Lloyd-Jones DM (2012). Competing cardiovascular outcomes associated with electrocardiographic left ventricular hypertrophy: the atherosclerosis risk in communities study. Heart 98: 330-334. DOI 10.1136/heartjnl-2011-300819.

Eizirik DL, Sammeth M, Bouckenooghe T, Bottu G, Sisino G, IgoilloEsteve M, Ortis F, Santin I, Colli ML, Barthson J, Bouwens L, Hughes L, Gregory L, Lunter G, Marselli L, Marchetti P, McCarthy MI, Cnop M (2012). The human pancreatic islet transcriptome: expression of candidate genes for type 1 diabetes and the impact of pro-inflammatory cytokines. PLoS Genetics 8: e1002552. DOI 10.1371/journal.pgen.1002552.

Foster M, Samman S (2010). Zinc and redox signaling: perturbations associated with cardiovascular disease and diabetes mellitus. Antioxidants \& Redox Signaling 13: 1549-1573. DOI 10.1089/ars.2010.3111.

Huang L, Teng T, Bian B, Yao W, Yu X, Wang X, Xu Z, Sun Y (2017). Zinc levels in left ventricular hypertrophy. Biological Trace Element Research 176: 48-55. DOI 10.1007/s12011-0160808-y.

Jang Y, Wang H, Xi J, Mueller RA, Norfleet EA, Xu Z (2007). NO mobilizes intracellular $\mathrm{Zn}^{2+}$ via cGMP/PKG signaling pathway and prevents mitochondrial oxidant damage in cardiomyocytes. Cardiovascular Research 75: 426-433. DOI 10.1016/j.cardiores.2007.05.015.

Kim I, Xu W, Reed JC (2008). Cell death and endoplasmic reticulum stress: disease relevance and therapeutic opportunities. Nature Reviews Drug Discovery 7: 1013-1030. DOI 10.1038/nrd2755.

Kim MH, Aydemir TB, Kim J, Cousins RJ (2017). Hepatic ZIP14mediated zinc transport is required for adaptation to endoplasmic reticulum stress. Proceeding of the National Academy of Sciences of the United States of America 114: E5805-E5814. DOI 10.1073/pnas.1704012114.

Lichten LA, Cousins RJ (2009). Mammalian zinc transporters: Nutritional and physiologic regulation. Annual Review of Nutrition 29: 153-176. DOI 10.1146/annurev-nutr-033009083312 .
Little PJ, Bhattacharya R, Moreyra AE, Korichneva IL (2010). Zinc and cardiovascular disease. Nutrition 26: 1050-1057. DOI 10.1016/j.nut.2010.03.007.

MacDonald RS (2000). The role of zinc in growth and cell proliferation. Journal of Nutrition 130: 1500S-1508S. DOI $10.1093 / \mathrm{jn} / 130.5 .1500 \mathrm{~S}$.

Murakami M, Hirano T (2008). Intracellular zinc homeostasis and zinc signaling. Cancer Science 99: 1515-1522. DOI 10.1111/ j.1349-7006.2008.00854.x.

Olgar Y, Durak A, Tuncay E, Bitirim CV, Ozcinar E, Inan MB, Tokcaer-Keskin Z, Akcali KC, Akar AR, Turan B (2018a). Increased free $\mathrm{Zn}^{2+}$ correlates induction of sarco(endo) plasmic reticulum stress via altered expression levels of $\mathrm{Zn}^{2+}$-transporters in heart failure. Journal of Cellular and Molecular Medicine 22: 1944-1956. DOI 10.1111/ jcmm.13480.

Olgar Y, Ozdemir S, Turan B (2018b). Induction of endoplasmic reticulum stress and changes in expression levels of $\mathrm{Zn}^{2+}$. transporters in hypertrophic rat heart. Molecular and Cellular Biochemistry 440: 209-219. DOI 10.1007/s11010017-3168-9.

Ron D, Walter P (2007). Signal integration in the endoplasmic reticulum unfolded protein response. Nature Reviews Molecular Cell Biology 8: 519-529. DOI 10.1038/nrm2199.

Roshanravan N, Alizadeh M, Hedayati M, Asghari-Jafarabadi M, Mesri Alamdari N, Anari F, Tarighat-Esfanjani A (2015). Effect of zinc supplementation on insulin resistance, energy and macronutrients intakes in pregnant women with impaired glucose tolerance. Iran Journal of Public Health 44: 211-217.

Rutkowski DT, Arnold SM, Miller CN, Wu J, Li J, Gunnison KM, Mori K, Sadighi Akha AA, Raden D, Kaufman RJ (2006). Adaptation to ER stress is mediated by differential stabilities of pro-survival and pro-apoptotic mRNAs and proteins. PLoS Biology 4: e374. DOI 10.1371/journal. pbio.0040374.

Ryul LS, Jin NS, Ryan PJ, Jeong YJ, Kim HK, Song IS, Xu Z, Kwon HY, Kang SC, Sohn EH, Ko KS, Rhee BD, Kim N, Han J (2015). The critical roles of zinc: beyond impact on myocardial signaling. Korean Journal of Physiology and Pharmacology 19: 389-399. DOI 10.4196/kjpp.2015.19.5.389.

Schroder M, Kaufman RJ (2005). ER stress and the unfolded protein response. Mutant Research 569: 29-63. DOI 10.1016/j. mrfmmm.2004.06.056.

Taylor KM, Morgan HE, Smart K, Zahari NM, Pumford S, Ellis IO, Robertson JFR, Nicholson RI (2007). The emerging role of the LIV-1 subfamily of zinc transporters in breast cancer. Molecular Medicine 13: 396-406. DOI 10.2119/2007-00040.Taylor.

Troche C, Aydemir TB, Cousins RJ (2016). Zinc transporter Slc39a14 regulates inflammatory signaling associated with hypertrophic adiposity. American Journal of Physiology, Endocrinology and Metabolism 310: E258-E268. DOI 10.1152/ajpendo.00421.2015.

Tuncay E, Bitirim VC, Durak A, Carrat GRJ, Taylor KM, Rutter GA, Turan B (2017). Hyperglycemia-induced changes in ZIP7 and $\mathrm{ZnT} 7$ expression cause $\mathrm{Zn}^{2+}$ release from the sarco (endo)plasmic reticulum and mediate ER stress in the heart. Diabetes 66: 1346-1358. DOI 10.2337/db16-1099.

Walter P, Ron D (2011). The unfolded protein response: from stress pathway to homeostatic regulation. Science 334: 1081-1086. DOI 10.1126/science.1209038.

Wang G, Huang H, Zheng H, He Y, Zhang Y, Xu Z, Zhang L, Xi J (2016). $\mathrm{Zn}^{2+}$ and mPTP mediate endoplasmic reticulum 
stress inhibition-induced cardioprotection against myocardial ischemia/reperfusion injury. Biological Trace Element Research 174: 189-197. DOI 10.1007/s12011-016-0707-2.

Xi J, Tian W, Zhang L, Jin Y, Xu Z (2010). Morphine prevents the mitochondrial permeability transition pore opening through $\mathrm{NO} / \mathrm{cGMP} / \mathrm{PKG} / \mathrm{Zn}^{2+} / \mathrm{GSK}$-3beta signal pathway in cardiomyocytes. AJP Heart and Circulatory Physiology 298: H601-H607. DOI 10.1152/ajpheart.00453.2009.

Xing F, Han H, He Y, Zhang Y, Jing L, Xu Z, Xi J (2017). Roles of endoplasmic reticulum stress in NECA-induced cardioprotection against ischemia/reperfusion injury. Oxidative Medicine and Cellular Longevity 2017: 1-10. DOI 10.1155/2017/2490501.

Yoshida H (2007). ER stress and diseases. FEBS Journal 274: 630658. DOI 10.1111/j.1742-4658.2007.05639.x.
Zhang D, Liu J, Tu H, Muelleman RL, Cornish KG, Li YL (2014a). In vivo transfection of manganese superoxide dismutase gene or nuclear factor kappa B shRNA in nodose ganglia improves aortic baroreceptor function in heart failure rats. Hypertension 63: 88-95. DOI 10.1161/HYPERTENSIONAHA.113.02057.

Zhang K (2010). Integration of ER stress, oxidative stress and the inflammatory response in health and disease. International Journal of Clinical and Experimental Medicine 3: 33-40.

Zhang X, Yuan Y, Jiang L, Zhang J, Gao J, Shen Z, Zheng Y, Deng T, Yan H, Li W, Hou WW, Lu J, Shen Y, Dai H, Hu WW, Zhang Z, Chen Z (2014b). Endoplasmic reticulum stress induced by tunicamycin and thapsigargin protects against transient ischemic brain injury: Involvement of PARK2-dependent mitophagy. Autophagy 10: 1801-1813. DOI 10.4161/auto.32136. 